\title{
Remote Monitoring and Control of Robotic Arm with Visual Feedback using Raspberry Pi
}

\author{
Ron Oommen Thomas \\ P.G. Student \\ Department of Electronics and Instrumentation \\ Engineering \\ Karunya University
}

\author{
K. Rajasekaran \\ Professor \\ Department of Electronics and Instrumentation \\ Engineering \\ Karunya University
}

\begin{abstract}
The common misconception regarding robots is that, they are used for industrial purpose only. But it is not so, they find applications in different fields, ranging from simple household chores to space explorations. There are different ways to control these robots, but imagine the case of controlling a robot miles and miles away through the internet. This paper describes the use of internet to control the robotic arm from a remote end by means of a visual feedback so the user can see the movement of the robotic arm from anywhere.
\end{abstract}

\section{General Terms}

Robotics, networking

\section{Keywords}

Raspberry Pi, robotic arm, web server, internet control.

\section{INTRODUCTION}

Telerobotics refers to control a robot from a remote end. With this, it is even possible to control the motion of the robot in space directly. Internet, satellites are some of the methods for teleoperation. The internet is universal and enables users to access any systems on the worldwide network cheaply. Considering the present trend and technologies, the tendency of electric home appliances is toward home networking. The implication is straightforward - one can control all household electric appliances and monitor the status from the remote end. With a web server, it becomes easy to control devices from a remote site. When such a web server is set up in a single board computer, it becomes a different story compared to other non OS based devices. Raspberry $\mathrm{Pi}$ is one such device. Others include Parallella (not yet released), OdroidX2, Hackberry, UDOO, APC Rock, Cubieboard, Beaglebone Black, Beagleboard,etc.

With the popularity and widespread use of internet, it becomes an easy task for anyone to control and monitor the robotic arm from internet[1-5]. Many spent more time in internet than the average time they sleep!! So with the introduction of an internet controlled robotic arm, the industrialists can even do their work from home and when used for household applications, the user can do it from his office or even from his tab, while driving!!

The Raspberry Pi is a credit-card-sized single-board computer developed in the UK by the Raspberry Pi Foundation. A USB camera (i-Ball) is used for visual feedback by providing live video streaming through the internet.

The Robotic Arm used is Maplin's OWI-535 Robotic Arm (non USB type). The Robotic arm can be controlled by a web server in the PC itself. Robotic arm control can be made wirelessly by means of Zigbee [6], [7] but can't be controlled from a remote site, so with a web server, it is possible. Another method for the control of robotic arm is by means of user motion like hand gesture control[8]. Internet based robots can be used for teleoperation, industry, automation, household applications. A web server is set up on the single board computer named Raspberry Pi. Many web server applications are available for the Raspberry Pi and Lightpd web server application was chosen to be the server for Raspberry Pi [9]. With the Pi set as a web server, it's possible for the client computers to access the data or web pages hosted by the Raspberry Pi server.

The Raspberry Pi has 17 GPIO pins. Using L293 motor driver boards, the Robotic Arm is controlled from the GPIO pins. A web page is hosted for the robotic arm control in the server.

\section{HARDWARE AND SOFTWARE: \\ 2.1 Raspberry Pi}

The Raspberry Pi [10] is based on a Broadcom BCM2835 system on a chip (SoC), which incorporates an ARM1176JZF-S $700 \mathrm{MHz}$ processor. The Raspberry Pi Foundation started off with a $256 \mathrm{MB}$ RAM, which was labeled as Model A, and later made a Model B with 512MB RAM. The GPU used is the VideoCore IV, owned by the Broadcom. It uses an SD card for booting and for memeory as it doesn't have an inbuilt hard disk for storage option. It was developed with the intention of teaching computer to school students but later turned out to be one of the prime choice for many applications and a within a span of 2 years, more than 1 millions have been sold. A supercomputer can be made out of Raspberry Pi by stacking 64 boards together.

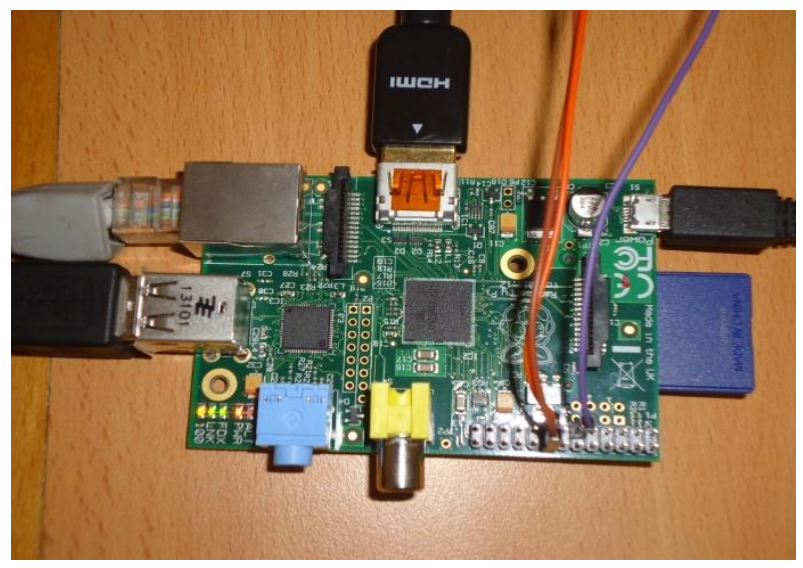

Figure 1: Raspberry Pi 


\subsection{Robotic Arm}

The Robotic arm used is the Maplin's OWI-535(non USB type) . It has four rotational joints- the base, shoulder, elbow and wrist. The gripper will open and close by means of the gear wheels. Except for the base which rotates the arm around the vertical z-axis, the other three rotate it around the $\mathrm{x}$-axis. The movement in the y-axis is absent in this arm which will limit the movement of the arm in whole. There is a limit to the movement each joint can produce.

\subsection{Web Camera}

The visual feedback is provided by the iBall 12.0 MP web camera. It has a still resolution of 12.0MP, video resolution of 2.0MP and a smooth video is given by $5 \mathrm{G}$ wide angle lens. A USB hub is used to interface camera with $\mathrm{Pi}$ because it is difficult for Pi to power it up after an USB keyboard and mouse are plugged-in.

\subsection{Motion}

Motion is a program that is used for the live video streaming. Where it monitors the video signal from the source camera and will detect changes i.e it can detect motion. Motion is a command line based tool. Graphical user interface is absent in motion. Everything is setup either via the command line or via configuration files.

\section{BLOCK DIAGRAM}

Here the Raspberry $\mathrm{Pi}$ is connected to the internet via LAN and the PC is connected to the internet via LAN or WIFI. The USB webcam provides the visual feedback necessary for monitoring the robotic arm from a remote end. Raspberry Pi needs an HDMI/VGA monitor for its display, which is here provided by the LCD projector through an HDMI connection. The robotic arm is controlled from the client side via the visual feedback.

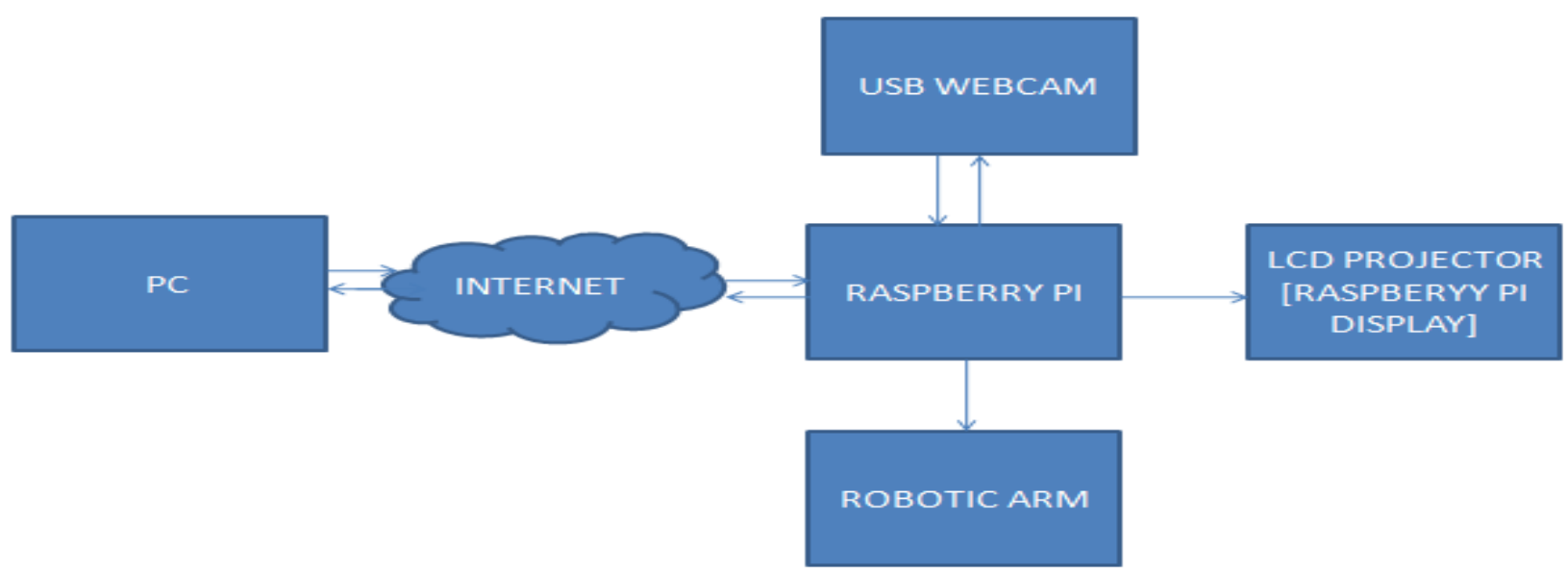

Figure 2: Block Diagram

\section{WEB SERVER}

The primary role of web servers is to deliver web content that can be accessed through the internet to the clients. Hypertext Transfer Protocol (HTTP) is the protocol that helps in the communication between client and server. Pages delivered are most frequently HTML documents which may include documents, images, style sheets and scripts in addition to text content. Web servers also support server-side scripting using Active Server Pages (ASP), PHP, or other scripting languages. Apache and Lighttpd are the most favored web server applications for Raspberry Pi.

\subsection{Raspberry Pi Web Server:}

Even though the $\mathrm{Pi}$ is significantly less powerful than most devices one would find in a data center, that doesn't mean that it can't act as a useful server in a home or business environment. Despite a small amount of memory and relatively underpowered processor, the $\mathrm{Pi}^{i}$ s low power draw and silent running makes it a great choice for serving lowtraffic simple pages to a local network or even out onto the Internet. A large proportion of modern web servers run a LAMP stack, a combination of Linux, Apache, MySQL and PHP. Different web server applications are available for
Raspberry Pi, like Lighttpd, Apache, Nginx, etc. It also has database support as a backend system for storing and retrieving data. Lighttpd having less footprint compared to Apache, is used as the web server in this setup. Lighttpd can be installed using the following command 'sudo apt-get $-y$ install Lighttpd'. Similarly, PHP is also installed in the system using commands. Once everything is installed, the web server must be restarted to take effect. The default directory for storing the web server files is /var/www. So whatever files are placed in this directory can be served upon request from the client. The directory is currently owned by the root user, so permission must be given to the web server user to access the files in the directory. The web server user for Lighttpd is 'www-data'.

\section{IMPLEMENTATION:}

\subsection{The Setup:}

At first, an operating system has to be set up in Raspberry Pi. The debian distribution was installed onto an SD card. The internal memory of Raspberry $\mathrm{Pi}$ is $512 \mathrm{MB}$, so an external storage memory is required for all the operations we do in the system, from storing a data to installing an application. As Raspberry $\mathrm{Pi}$ is a single board computer, we need a mouse and 
keyboard to work, with the 2 USB ports available, it's possible to connect the mouse and keyboard to the system. If another USB device has to be connected to Raspberry Pi, a powered USB hub should be used because the device will draw power from the Pi to work and this will cause problems like automatic shutdown or the Pi will hang. Even though the external powered hub will provide the necessary current for Pi to operate, it will be good to use an external adapter. After the initial boot up, configurations have to be changed to suit our needs like initial boot up to the desktop, etc. After connecting to a network via LAN, we need to check for the IPV4-address assigned to the system using the command 'sudo ifconfig'. As this is the dynamic IP address, it's possible to change it to a static IP by editing the interface file present in /etc file. Then update the software and kernel as required to make the system up to date.

The video streaming can be enabled by using the motion software and the configuration file has to be edited in order to suit our needs for live streaming. The default port through which the video will be out is port 81 .

Using a suitable editor, the web page has to be designed. A dynamic web page is created and is saved in the web server default directory /var/www. Python is the preferred language for Raspberry Pi, but it also supports C, Java, Ruby, etc.. Python programs are written for the forward and backward motion of each joint of the arm and are linked with the PHP script. The entire experimental setup is shown in figure 3 .

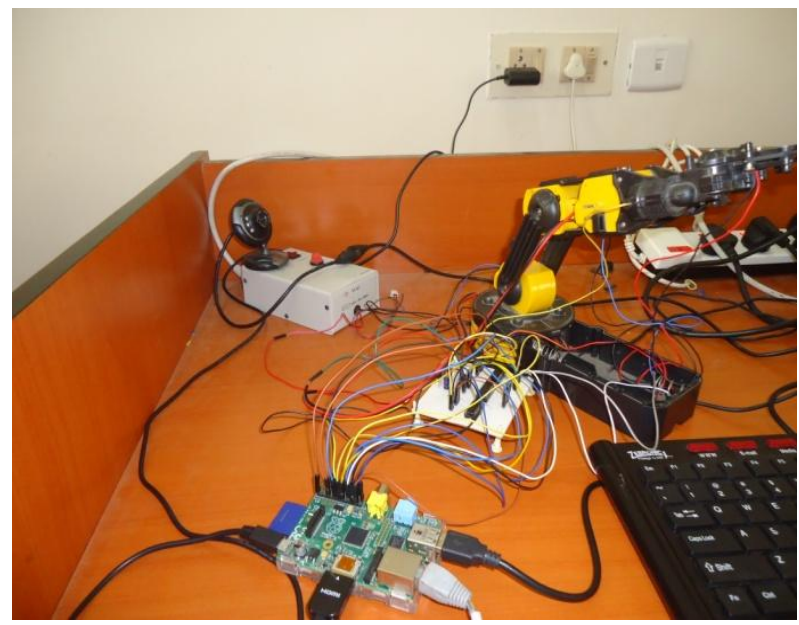

Figure 3: The experimental setup

The interfacing of the motors of the robotic arm with the Raspberry $\mathrm{Pi}$ is shown in the figure 4 . The robotic arm has 5 motors, so 3 motor driver ICs are required for the control of all the joints of the robotic arm. A triple L293 driver board which consists of three L293D motor driver ICs is designed for the control of all the motors and is interfaced with the GPIO pins of Raspberry Pi. The enable pin should be made high for the motor to run and by changing the values of the input pins, we can change the direction of motion of the arm. Each driver IC can control a maximum of two motors. A total of 15 GPIO pins of Raspberry Pi are used for the control of all the joints of robotic arm. For a motor driver IC like L293D, we need to provide separate power supply for both the IC and motors connected to it. As all the motors of the arm works at $5 \mathrm{~V}$, a single $5 \mathrm{~V}$ supply is given for both the IC and the motor.

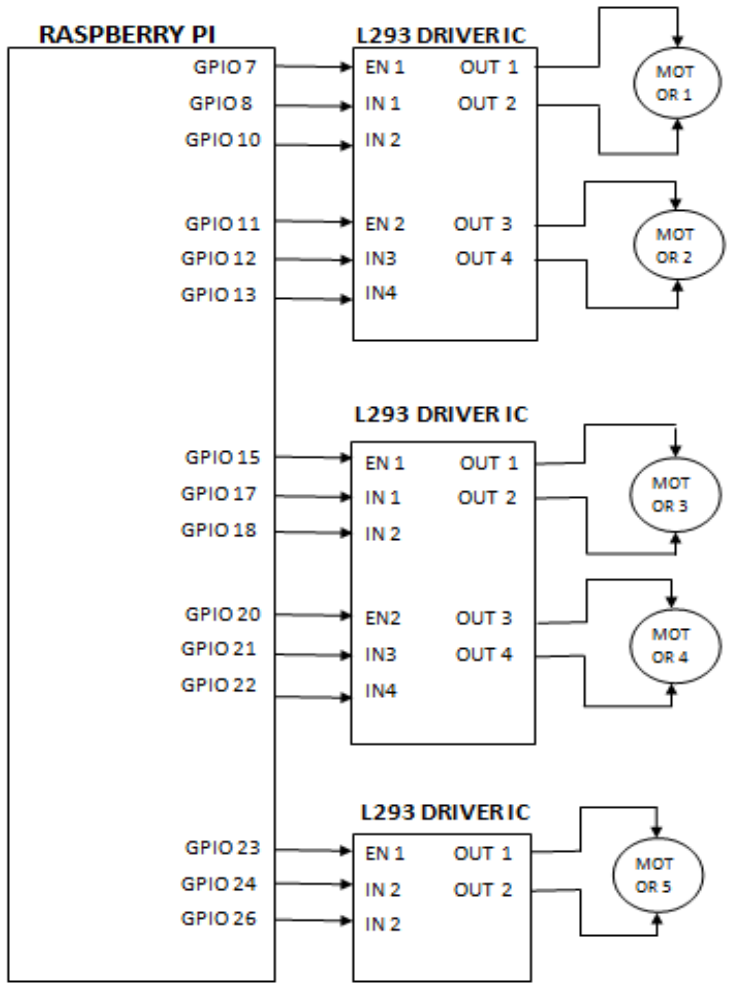

Figure 4: Raspberry Pi connection to the DC motor of robotic arm through driver ICs

\section{IMPLEMENTATION:}

\subsection{Live Video Streaming}

Video streaming is an integral part of this project as it serves as the means by which the user can monitor the robotic arm from a remote end, otherwise the user has no other way to determine whether the arm is moving or not, unless he is present at the local site where the arm is present. The live video streaming is shown in the Figure 5. The video is out through the port 81 and can be viewed from any browser by providing the IP address in the address bar of the client browser - 192.168.1.2:8081

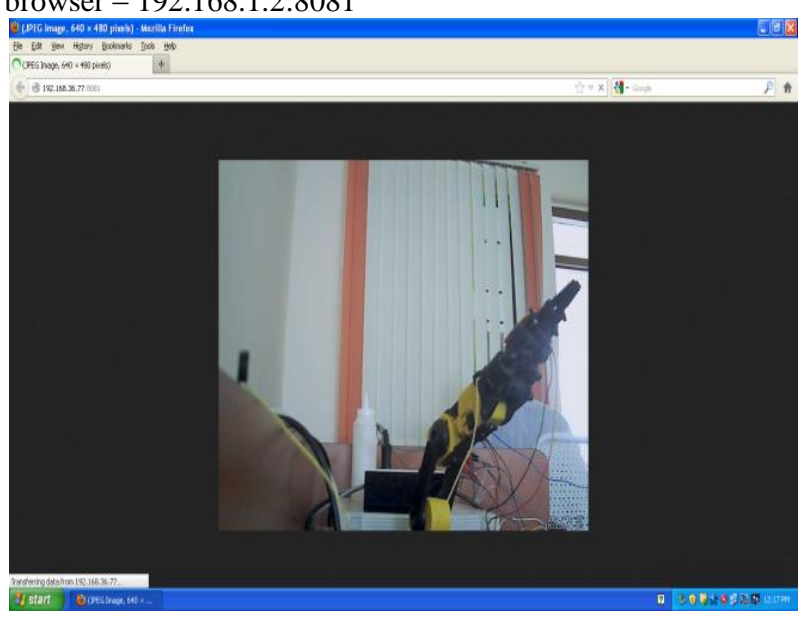

Figure 5: Live video streaming through port 81

\subsection{Robotic Arm Control with Live Video Streaming}

Once the video streaming is working perfectly, a web page is designed to integrate the video streaming with the buttons to control the arm. As there are 5 motors for the arm, 5 pair of 
buttons are created for the arm movement in both forward and backward direction. Buttons are created using Ajax and PHP is used for the button controls such that each press will execute the corresponding python program at the server side. The reason why Ajax is used is that for each button press, the web page will reload and the video will also reload and is out for atleast 2 seconds. At that time the user willn't be able to see the movement of robotic arm. When the buttons are created using Ajax, the web page will not reload and the user can see the live streaming as it is. The Figure 6 shows the web page designed for robotic arm control and live video streaming. The address for the web page is 192.168.1.2/RoboticArm.php

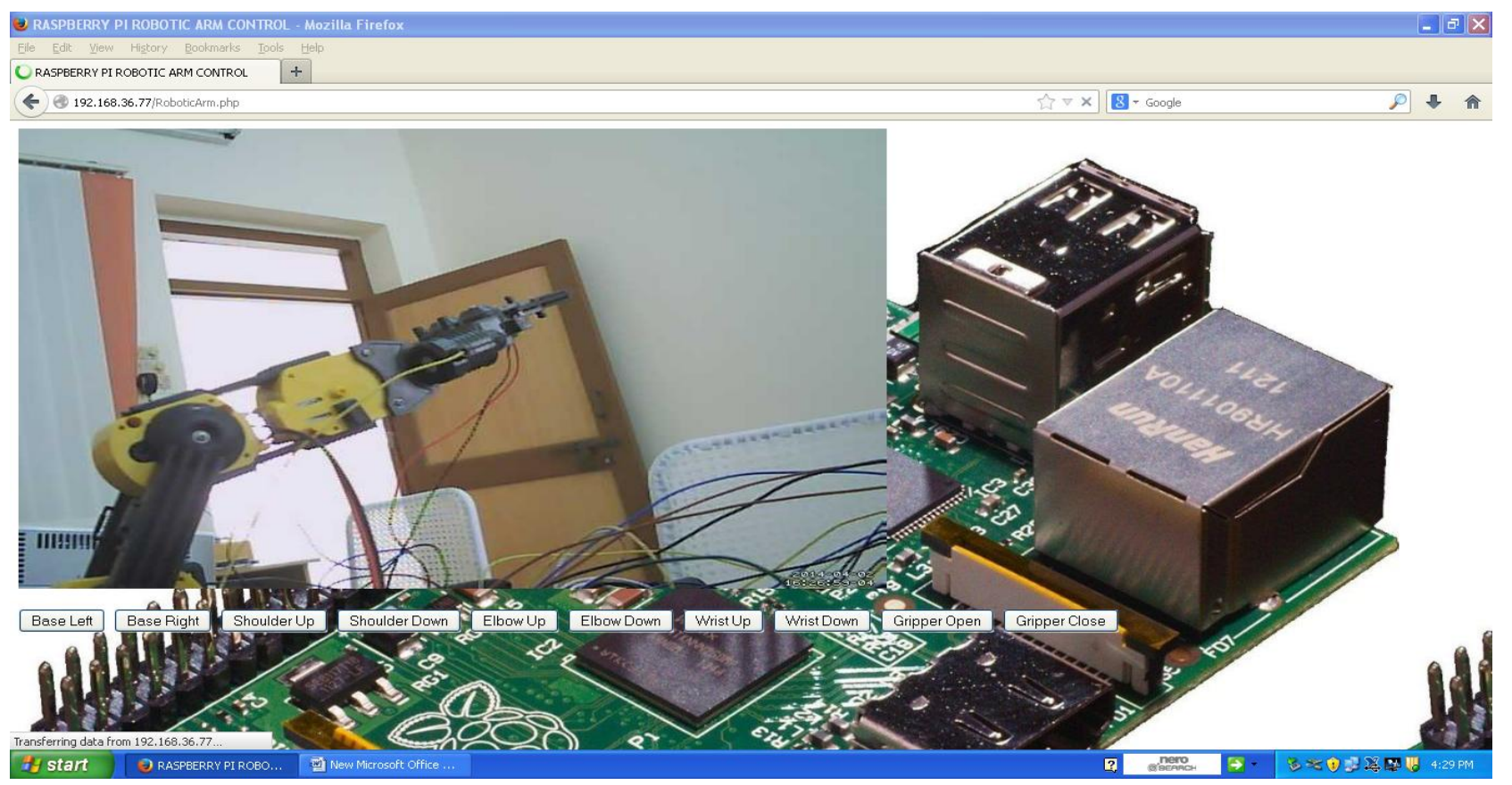

Figure 6: Web page designed for robotic arm control and live video streaming

\section{CONCLUSION AND FUTURE WORK:}

The purpose of this project isto control the robotic arm from internet with live video streaming as a means of visual feedback. This project is almost same as [2] with the advantage that with port forwarding it is possible for a person in a different country to see the robotic arm and control it according to his needs, whereas many other projects require user to be at the station as there is no means of giving a visual feedback. In future, by making a mobile robot with a robotic arm by means of a wireless network, it can be made a multipurpose robot, ranging from surveillance and home security to industrial applications where the user need not be present at the work place in person but can do it from his home itself.

\section{ACKNOWLEDGEMENT}

I express my sincere gratitude to my guide Prof. K. Rajasekaran who has supported me throughout this project.

\section{REFERENCES}

[1] V. Ramya, B. Palaniappan, T. Akilan "Embedded System for Robotic Arm Movement Control using Web Server and ZigBee Communication", International Conference on Research Trends in Computer Technologies, Proceedings published in International Journal of Computer Applications ${ }^{\circledR}$ (IJCA) (0975 8887), pg 30-34, 2013

[2] Wan MuhamadHanif Wan Kadira, Reza EzuanSaminb, Babul Salam Kader Ibrahim C. "Internet Controlled Robotic Arm", Science Direct, International Symposium on Robotics and Intelligent Sensors , pg 1065-1071, 2012.
[3] Lung Ngai, Wyatt S. Newman, Vincenzo Liberatore, "An Experiment in Internet-Based, Human-Assisted Robotics", Case Western Reserve University, pg-10111015,IEEE, 2002.

[4] Huosheng Hu, Lixiang Yu, PuiWoTsui, Quan "Internetbased Robotic Systems for Teleoperation" International Journal of Assembly Automation, Vol. 21, No. 2, pg 110,2000

[5] Kuk-Hyun Han, Yong-Jae Kim, Jong-Hwan Kim and Steve Hsia, "Internet Control of Personal Robot between KAIST and UC Davis", 2000.

[6] R.A. Kadu, Prof. V.A. More, P.P. Chitte, J.G. Rana, M.R. Bendre . "Wireless Control \& Monitoring of Robotic Arm" International Journal of Computer Technology and Electronics Engineering (IJCTEE) Volume 2, Issue 1, pg 28-38, 2010

[7] Christian Hernández, RacielPoot, Lizzie Narváez, Erika Llanes and Victor Chi "Design and Implementation of a System for Wireless Control of a Robot" International Journal of Computer Science Issues, Vol. 7, Issue 5, pg 191-197, September 2010

[8] Heng-Tze Cheng, Zheng Sun, Pei Zhang, "Real-Time Imitative Robotic Arm Control for Home Robot Applications", Carnegie Mellon University, IEEE, March 2011

[9] Lighttpd Web Server http://www.lighttpd.net

[10] Raspberry Pi Forum and Discussion page http:// www.raspberrypi.org/phpBB3. 